Tara K. lyer, MD, NCMP

Clinical Fellow in Women's Health, Department

of Obstetrics and Gynecology, Center for

Specialized Women's Health, Cleveland Clinic,

Cleveland, $\mathrm{OH}$
Heather Hirsch, MD, MS, NCMP

Clinical Program Director, Menopause and

Midlife Clinic; Clinical Instructor of Medicine,

Division of Women's Health, Brigham and

Women's Hospital, Harvard Medical School

Boston, MA

\title{
Clinical impact of 2020 American Heart Association statement on menopause and cardiovascular disease risk
}

\section{ABSTRACT}

The American Heart Association published a 2020 scientific statement on cardiovascular disease risk for women transitioning through or experiencing menopause. The report reflects scientific evidence on menopause and cardiovascular risks, and this article reviews the statement with a focus on what is new and what is clinically important for healthcare providers treating this patient population.

\section{KEY POINTS}

The American Heart Association 2020 scientific statement supports the notion that the transition of menopause itself, independent of chronological and ovarian aging, leads to increased cardiovascular risk and mortality.

The physiological changes that take place during the female reproductive life span (extreme early age at menarche, pregnancy disorders, preterm births, adverse pregnancy outcomes, early age at menopause, and menopause) are associated with cardiometabolic risk.

Female patients in midlife should be routinely counseled on lifestyle interventions aimed at achieving ideal cardiovascular health: smoking cessation, weight management, optimization of cholesterol levels and blood pressure and fasting blood glucose, daily physical activity, and dietary approaches to stop hypertension.

\begin{abstract}
Cardiovascular disease (CVD) is the lead$C$ ing cause of mortality for women worldwide. ${ }^{1,2}$ Women typically develop CVD several years later than men, although "this female advantage gradually disappears with aging, particularly after menopause when cardiometabolic risk factors accumulate." 3 The physiological changes that take place during the different stages of the female reproductive life span, such as extreme early age at menarche, pregnancy disorders, preterm births and adverse pregnancy outcomes, early age at menopause ( $<40$ years), and menopause, are factors associated with cardiometabolic risk. ${ }^{2,4,5}$

In December 2020, the American Heart Association (AHA) published a scientific statement focused on menopause and CVD risk with the intent of increasing awareness of adverse cardiometabolic health-related changes accompanying female patients midlife. ${ }^{1} \mathrm{Ad}$ verse changes in body fat distribution, lipids, lipoproteins, and structural and functional measures of vascular health take place during the transition to menopause, seen as a time of accelerating CVD risk. ${ }^{1,6}$
\end{abstract}

\section{CLINICAL SETTING}

The field of menopause is continuously evolving as studies are completed regarding best treatment options for female patients dealing with menopause-related symptoms and risk for CVD. This review focuses on the 2020 AHA scientific statement for the purpose of raising 
clinician awareness of the significant adverse cardiometabolic health-related changes and mitigation of CVD risk in this patient population. ${ }^{1}$

\section{INTENDED AUDIENCE}

This review is intended for healthcare providers who manage female patients in the middle of their lives transitioning to or experiencing menopause in the outpatient setting including the primary care, cardiovascular, endocrinology, and gynecology environments.

\section{WHO WROTE THE GUIDELINES?}

The guidelines were completed by the American Heart Association Prevention Science Committee of the Council on Epidemiology and Prevention and the Council on Cardiovascular and Stroke Nursing, composed of a collaboration of physicians and researchers. The documents were peer-reviewed by the American Heart Association Office of Science Operations.

\section{WHAT ARE THE MAIN RECOMMENDATIONS?}

Menopause, The December 2020 AHA scientific statement independent of chronological and ovarian aging, leads to increased cardiovascular risk and mortality reviewed longitudinal research supporting the notion that the transition of menopause itself, independent of chronological and ovarian aging, leads to increased cardiovascular risk and mortality. ${ }^{1}$ Previous AHA guidelines for the prevention of CVD recommended that female patients in midlife should be evaluated for potential contributors to poor cardiovascular health (ie, smoking, overweight or obesity, hypertension, diabetes, etc.), stratified into categories of risk (ie, ideal cardiovascular health, at risk, and high risk), and then counseled accordingly., ${ }^{7,8}$ While previous studies have noted that premature menopause $(<40$ years old) and early menopause (40 to 45 years old) can increase the risk of CVD, clinicians should now also recognize menopause in general as a risk factor. ${ }^{1-5}$

The clinical recommendations highlighted in the scientific statement focus on the role of lifestyle intervention, lipid-lowering medications, and menopausal hormone therapy (HT) in the prevention of CVD during the critical window of midlife of the female patient. ${ }^{1}$

\section{Lifestyle interventions}

Female patients traversing menopause in midlife should be routinely counseled on lifestyle interventions aimed at achieving ideal cardiovascular health. In accordance with the AHA Life's Simple 7 components, ${ }^{1,9}$ to achieve ideal cardiovascular health, interventions should involve the following:

- Smoking cessation

- Weight management to achieve an ideal body weight (body mass index $<25 \mathrm{~kg} / \mathrm{m}^{2}$ )

- Optimization of cholesterol levels: total cholesterol $<200 \mathrm{mg} / \mathrm{dL}$, low density lipoprotein cholesterol (LDL-C) $<100 \mathrm{mg} / \mathrm{dL}$, high density lipoprotein cholesterol (HDLC) $>50 \mathrm{mg} / \mathrm{dL}$, triglycerides $<150 \mathrm{mg} / \mathrm{dL}$, non-HDL-C $<130 \mathrm{mg} / \mathrm{dL}$ )

- Optimization of blood pressure levels $(<120 / 80 \mathrm{mmHg})$

- Optimization of fasting blood glucose (goal $<100 \mathrm{mg} / \mathrm{dL}$ )

- Engaging in at least $150 \mathrm{~min} /$ week of moderate-intensity exercise or 75 minutes per week of vigorous exercise (or a combination of both)

- A DASH (Dietary Approaches to Stop Hypertension) diet. ${ }^{1,7-9}$

\section{Aspirin use}

While not discussed in detail in this scientific statement, previous AHA guidelines have outlined that aspirin use in female patients without cardiac risk factors and under the age of 65 is not recommended for primary prevention of heart attack. ${ }^{7,8}$

\section{Lipid-lowering interventions}

First-line intervention strategies for the optimization of lipids in midlife female patients include lifestyle modifications such as regular exercise, maintaining ideal body weight, smoking abstinence, and eating a hearthealthy diet. ${ }^{1,7,8}$

It is unclear whether supplementation and/or dietary intake of foods rich in omega-3 and omega- 6 fatty acids are associated with reduced coronary heart disease, myocardial infarction, reduced total cholesterol levels, or decreased rates of cardiovascular or all-cause mortality, leaving it uncertain what effects, if any, omega- 3 and omega- 6 fatty acids have on CVD prevention in midlife women. ${ }^{7}$ Con- 
sumption of omega-3 fatty acids in the form of fish or in capsule form (eg, eicosapentaenoic acid $1800 \mathrm{mg} / \mathrm{d}$ ) may be considered in women with hypercholesterolemia and/or hypertriglyceridemia for primary and secondary prevention. ${ }^{?}$

The role of 3-hydroxy-3-methylglutaryl coenzyme A (HMG-CoA) reductase inhibitors (ie, statins) for CVD risk reduction in women in midlife remains controversial. Current guidelines still recommend statins as first-line therapy for risk reduction of CVD in patients with LDL-C $\geq 190 \mathrm{mg} / \mathrm{dL}$, diabetes, and patients 40 to 75 years old at sufficiently elevated CVD risk. ${ }^{1,8}$ However, the literature surrounding these recommendations is conflicting and not well-differentiated by gender. While there have been several randomized controlled trials demonstrating reduced atherosclerotic CVD risk in patients using statin therapy for primary prevention in mixed gender studies, there have also been multiple studies that have not shown significant reductions in women specifically. ${ }^{10-16}$ The Justification for Use of statins in Prevention: an Intervention Trial Evaluating Rosuvastatin (JUPITER) trial demonstrated a reduction in arterial revascularization in a subgroup of female patients $>60$ years old, but did not find a statistically significant decrease in myocardial infarction or all-cause mortality with statin use across the entire female cohort. ${ }^{1,10}$ In the Management of Elevated Cholesterol in the Primary Prevention Groups of Adult Japanese (MEGA) study that followed a large female cohort for $>5$ years of statin therapy, the impact on CVD and all-cause mortality was null. ${ }^{1,11}$ The Heart Outcomes Prevention Evaluation-3 (HOPE-3) trial also looked at sex-specific data and found that statin therapy had a nonsignificant effect on CVD and all-cause mortality. ${ }^{1,12}$ Multiple meta-analyses found mixed results regarding lipid lowering medications affecting prevention of total or CVD mortality or risk factors in general; for female patients with known CVD, medications reduced cardiovascular events and mortality as well as nonfatal myocardial infarction but did not impact total mortality. ${ }^{13-16}$

The AHA scientific statement noted that data for primary and secondary prevention and improved survival with lipid-lowering medications in women remains elusive, and nonpharmacological therapies are currently first-line strategies for improving lipid profiles. ${ }^{1}$ Additionally, given diabetes is a known risk factor for CVD, it is important to note that there have been studies suggesting an increased risk of diabetes associated with statin use in postmenopausal female patients. ${ }^{17}$

The most common nonstatin therapies are bile acid sequestrants, cholesterol absorption inhibitors, and proprotein convertase subtili$\sin /$ kexin type 9 inhibitors that may be used in addition to or instead of statins for cholesterol reduction in select patients. ${ }^{1,7,8}$ The use of these medications in the primary prevention of CVD in both men and women is still in question and warrants sex-specific trials to determine efficacy.

\section{Supplements}

There are currently no vitamin or antioxidant supplements recommended for primary or secondary prevention of CVD. ${ }^{1,7,8}$

\section{Menopausal hormone therapy}

Currently, no data are available on the effects of menopausal HT on the cardiometabolic health of perimenopausal women. ${ }^{1}$ Evidence varies regarding the effects of HT on atherosclerosis and CVD event progression by age and timing of HT initiation. ${ }^{1}$ The use of HT and selective estrogen receptor modulators for primary or secondary prevention of CVD and stroke in postmenopausal women is not currently recommended. ${ }^{1}$ However, according to the AHA scientific statement, post-hoc and longitudinal research suggests that HT initiated early among patients with premature or surgical menopause and within 10 years of natural menopause is associated with cardiovascular benefit. ${ }^{1}$ Additionally, the use of hormone replacement therapy to mitigate certain deleterious effects of early estrogen loss is currently considered the standard of care. ${ }^{18}$

\section{WHAT IS DIFFERENT FROM PREVIOUS GUIDELINES?}

The most paramount distinction introduced in the 2020 AHA scientific statement is the identification of the menopause transition as a risk factor for CVD. ${ }^{1}$ The 2011 AHA Guidelines did not classify menopause, early meno-

\section{Significant adverse cardiometabolic changes occur in women in midlife}


pause, or surgical menopause as a risk factor for CVD, nor did it address menopause and its cardiometabolic significance. ${ }^{7}$ While the 2019 AHA guidelines on primary prevention of CVD identify early menopause as a possible risk factor for CVD, it does not address the consequences of natural menopause. ${ }^{8}$

\section{DO OTHER SOCIETIES AGREE OR DISAGREE?}

The current AHA scientific statement stands in agreement with multiple other national medical societies in the field of menopause, including the North American Menopause Society, the American College of Obstetricians and Gynecologists, the Endocrine Society, the American College of Endocrinology, and the American Association of Clinical Endocrinologists. ${ }^{18-20}$ These prominent organizations recognize the cardiometabolic changes of menopause as a risk factor for CVD and suggest that for healthy, recently menopausal patients, the benefits of HT outweigh the risks. ${ }^{18-20}$ In congruence with the AHA, none of these societies currently recommend the use of HT for primary prevention of CVD. ${ }^{18-20}$ The North American Menopause Society does, however, recommend the use of HT, to mitigate physiologic consequences of early estrogen loss, until at least the median age of menopause ( 52 years old) in patients without contraindications who undergo menopause early or prematurely. ${ }^{18}$ All of these societies endorse lifestyle modifications and statin therapy, when appropriate, for reduction of CVD risk in midlife women. ${ }^{18-20}$

\section{HOW WILL THIS CHANGE DAILY PRACTICE?}

The 2020 AHA scientific statement identifies a crucial gap in the healthcare of female patients in midlife. ${ }^{1}$ Currently, there is a widespread missed opportunity to educate women about the health risks associated with menopause. The AHA recommends that female patients be routinely counseled on lifestyle interventions aimed at achieving ideal cardiovascular health and suggests clinicians explain to patients that these lifestyle modifications are important to help counteract the consequences of the estrogen loss that occurs at the menopausal transition. ${ }^{1}$

There is also a current critical unmet need for individualized counseling on the indications and benefits of HT as a treatment option for menopausal women. The ramifications of menopausal HT on the cardiovascular health of female patients has been a source of great debate among healthcare providers in recent decades and first arose in 2002, when the Women's Health Initiative published its initial results suggesting an increased risk of cardiovascular events among postmenopausal patients treated with HT. ${ }^{21}$ Post-hoc analyses of the data and other subsequent studies showed no risk or a reduction in cardiovascular risk when HT was initiated at or before the age of 60 , and/or within 10 years of the last menstrual period. ${ }^{22-25}$ Despite this, controversy around the safety of HT has remained prevalent, resulting in a sharp decline in the number of HT prescriptions written since $2003 .{ }^{26}$ For this reason, the 2020 AHA scientific statement regarding cardiometabolic changes and CVD risk throughout the transition into menopause will be an important milestone in the healthcare of female patients in midlife. ${ }^{1}$ This is a significant clinical consideration because the development of chronic disease states are commonly diagnosed around the menopause transition, often coinciding with the time that patients are seeking medical treatment for menopausal symptoms.

The updated position of the AHA on menopause represents a turning point in the healthcare of female patients that should not be underestimated. The scientific statement published by the AHA will hopefully go a long way in facilitating the dissemination and understanding of decades of evidence-based research regarding the treatment of menopause.

\section{WHEN WOULD GUIDELINES NOT APPLY?}

The AHA recommendations to recognize the cardiometabolic changes during the transition to menopause, menopause, and the midlife of women as important risk factors for CVD and mortality would apply to all patients who lose ovarian hormone production, regardless of when this loss occurs. Guidelines on the use of HT would not apply to patients with possible contraindications to estrogen therapy, such as medical history of unexplained vaginal bleeding, severe active liver disease, porphyria 
cutanea tarda, estrogen-sensitive malignancy, stroke, pulmonary embolism, deep vein thrombosis, dementia, and unstable coronary heart disease.

\section{REFERENCES}

1. El Khoudary SR, Aggarwal B, Beckie TM, et al; on behalf of the American Heart Association Prevention Science Committee of the Council on Epidemiology and Prevention; and Council on Cardiovascular and Stroke Nursing. Menopause transition and cardiovascular disease risk: implications for timing of early prevention: a scientific statement from the American Heart Association. Circulation 2020; 142(25):e506-e532. doi:10.1161/CIR.0000000000000912

2. Benjamin EJ, Muntner $P$, Alonso A, et al; on behalf of the American Heart Association Council on Epidemiology and Prevention Statistics Committee and Stroke Statistics Subcommittee. Heart disease and stroke statistics-2019 update: A report from the American Heart Association. Circulation 2019; 139(10):e56-e528. doi:10.1161/CIR.0000000000000659

3. Roa-Diaz ZM, Raguindin PF, Bano A, Laine JE, Muka T, Glisic M. Menopause and cardiometabolic diseases: what we (don't) know and why it matters. Maturitis 2021; 152:48-56. doi:10.1016/j.maturitas.2021.06.013

4. Peltier M, Trojette F, Sarano ME, Grigioni F, Slama MA, Tribouilloy CM. Relation between cardiovascular risk factors and nonrheumatic severe calcific aortic stenosis among patients with a three-cuspid aortic valve. Am J Cardiol 2003; 91(1):97-99. doi:10.1016/s0002-9149(02)03010-2

5. Martinsson A, Li X, Zoller B, Andell P, Andersson C, Sundquist K, Smith JG. Familial aggregation of aortic valvular stenosis: a nationwide study of sibling risk. Circ Cardiovasc Genet 2017: 10(6):e001742. doi:10.1161/circgenetics.117.001742

6. El Khoudary SR. Gaps, limitations and new insights on endogenous estrogen and follicle stimulating hormone as related to risk of cardiovascular disease in women traversing the menopause: a narrative review. Maturitas 2017; 104:44-53. doi:10.1016/j.maturitas.2017.08.003

7. Mosca L, Benjamin EJ, Berra K, et al. Effectiveness-based guidelines for the prevention of cardiovascular disease in women-2011 update: a guideline from the American Heart Association. Circulation 2011; 123(11):1243-1262. doi:10.1161/CIR.0b013e31820faaf8

8. Arnett DK, Blumenthal RS, Albert MA, et al. 2019 ACC/AHA guideline on the primary prevention of cardiovascular disease: a report of the American College of Cardiology/American Heart Association Task Force on Clinical Practice Guidelines. Circulation 2019; 140(11):e596-e646. doi:10.1161/CIR.0000000000000678

9. Lloyd-Jones DM, Hong Y, Labarthe D, et al; on behalf of the American Heart Association Strategic Planning Task Force and Statistics Committee. Defining and setting national goals for cardiovascular health promotion and disease reduction: the American Heart Association's strategic Impact Goal through 2020 and beyond. Circulation 2010; 121(4):586-613. doi:10.1161/CIRCULATIONAHA.109.192703

10. Ridker PM, Pradhan A, MacFadyen JG, Libby P, Glynn RJ. Cardiovascular benefits and diabetes risks of statin therapy in primary prevention: an analysis from the JUPITER trial. Lancet 2012; 380(9841):565571. doi:10.1016/S0140-6736(12)61190-8

11. Mizuno K, Nakaya N, Ohashi Y, et al. Usefulness of pravastatin in primary prevention of cardiovascular events in women: analysis of the Management of Elevated Cholesterol in the Primary Prevention Group of Adult Japanese (MEGA study). Circulation 2008; 117(4):494-502. doi:10.1161/CIRCULATIONAHA.106.671826

12. Yusuf S, Bosch J, Dagenais G, et al; HOPE-3 Investigators. Cholesterol lowering in intermediate-risk persons without cardiovascular disease. N Engl J Med 2016; 374:2021-2031. doi:10.1056/NEJMoa1600176

13. Petretta M, Costanzo P, Perrone-Filardi P, Chiariello M. Impact

\section{DISCLOSURES}

The authors report no relevant financial relationships which, in the context of their contributions, could be perceived as a potential conflict of interest.

of gender in primary prevention of coronary heart disease with statin therapy: a meta-analysis. Int J Cardiol 2010; 138(1):25-31. doi:10.1016/j.ijcard.2008.08.001

14. Brugts JJ, Yetgin T, Hoeks SE, et al. The benefits of statins in people without established cardiovascular disease but with cardiovascular risk factors: meta-analysis of randomised controlled trials. BMJ 2009; 338:b2376. doi:10.1136/bmj.b2376

15. Cholesterol Treatment Trialists' (CTT) Collaboration. Efficacy and safety of LDL-lowering therapy among men and women: meta-analysis of individual data from 174000 participants in 27 randomised trials. Lancet 2015; 385(9976):1397-1405. doi:10.1016/S0140-6736(14)61368-4

16. Walsh JM, Pignone M. Drug treatment of hyperlipidemia in women. JAMA 2004; 291(18):2243-2252. doi:10.1001/jama.291.18.2243

17. Culver AL, Ockene IS, Balasubramanian R, et al. Statin use and risk of diabetes mellitus in postmenopausal women in the Women's Health Initiative. Arch Intern Med 2012; 172(2):144-152. doi:10.1001/archinternmed.2011.625

18. The North American Menopause Society 2017 Hormone Therapy Position Statement Advisory Panel. The 2017 hormone therapy position statement of The North American Menopause Society. Menopause 2017; 24(7):728-753. doi:10.1097/GME.0000000000000921

19. The American College of Obstetricians and Gynecologists Committee Opinion No. 565: Hormone therapy and heart disease. Obstet Gynecol 2013; 121(6):1407-1410. doi:10.1097/01.AOG.0000431053.33593.2d

20. Jellinger PS, Handelsman Y, Rosenblit PD, et al. American Association of Clinical Endocrinologists and American College of Endocrinology guidelines for management of dyslipidemia and prevention of cardiovascular disease. Endocr Pract 2017; 23(suppl 2):1-87. doi:10.4158/EP171764.APPGL

21. Writing Group for the Women's Health Initiative Investigators. Risks and benefits of estrogen plus progestin in healthy postmenopausal women: principal results from the Women's Health Initiative randomized controlled trial. JAMA 2002; 288(3):321-333. doi:10.1001/jama.288.3.321

22. Manson JE, Chlebowski RT, Stefanick ML, et al. Menopausal hormone therapy and health outcomes during the intervention and extended poststopping phases of the Women's Health Initiative randomized trials. JAMA 2013; 310(13):1353-1368. doi:10.1001/jama.2013.278040

23. Manson JE, Aragaki AK, Rossouw JE, et al. Menopausal hormone therapy and long-term all-cause and cause-specific mortality: the Women's Health Initiative randomized trials. JAMA 2017; 318(10):927-938. doi:10.1001/jama.2017.11217

24. Mikkola TS, Tuomikoski $\mathrm{P}$, Lyytinen $\mathrm{H}$, et al. Estradiol-based postmenopausal hormone therapy and risk of cardiovascular and all-cause mortality. Menopause 2015; 22(9):976-983. doi:10.1097/GME.0000000000000450

25. Hodis HN, Mack WJ. In perspective: estrogen therapy proves to safely and effectively reduce total mortality and coronary heart disease in recently postmenopausal women. Menopause Manag 2008; 17(2):27-32. pmid:20490363

26. Crawford SL, Crandall CJ, Derby CA, et al. Menopausal hormone therapy trends before versus after 2002: impact of the Women's Health Initiative study results. Menopause 2018; 26(6):588-597. doi:10.1097/GME.0000000000001282

Address: Tara K. lyer, MD, NCMP, Clinical Fellow in Women's Health, Department of Obstetrics and Gynecology, Center for Specialized Women's Health, A10, Cleveland Clinic, 9500 Euclid Avenue, Cleveland, OH 44195; iyert@ccf.org 